\title{
Proceedings of the winter meeting of the British Neuropsychiatry Association, London Zoo, Regent's Park, London, 17 January 1997
}

The topic of the meeting was memory. The morning session was devoted to theoretical and experimental aspects. Professor Alan Parkin (Sussex) discussed theories of amnesia and confabulation with special reference to patients with frontal lobe damage in whom confabulation, source amnesia, and impairment of metamemory and memory for temporal order may have a common basis. His work using a false recognition paradigm suggests that encoding and retrieval deficits can contribute to the generation of false alarm rates. A broadening of attention with difficulty in focusing on a central event may be the cause of this phenomenon which is likely to be relevant in patients exhibiting confabulation, although the presence of other frontal lobe deficits may also be necessary.

Dr Karalyn Patterson (Cambridge) discussed semantic memory and its disorders. Semantic memory (conceptual knowledge) is impaired in Alzheimer's disease, but only in the advanced stages. The term semantic dementia is used to describe temporal lobe type of dementia, such as Pick's disease, in which impairment of semantic memory may be the only deficit for several years before a more pervasive cognitive impairment supervenes. Loss of semantic memory impinges on other functions and results in loss of vocabulary and knowledge about objects and people, whereas aspects of episodic memory, syntax, and phonology are spared. Data from longitudinal studies suggest that impairment of specific semantic memory becomes blurred as discrimination tasks become more difficult and that naming and language production and comprehension deteriorate with progression of disease.

Dr Simon Fleminger (London) reviewed the confusing terminology surrounding the symptom of reduplicative paramnesia, which has been considered as a form of delusional misidentification, as a phenomenon closely related to confabulation or delusional memory and as a form of temporospatial disorientation. Errors in the temporal and context binding of memories seem to be common to reduplicative paramnesia and confabulation, but in reduplicative paramnesia loss of perceptual and topographical orientation may also be relevant. This suggests that in addition to frontal lobe damage, right hemispheric dysfunction may also need to be present for reduplicative paramnesia to appear.

Professor R Dolan (London) discussed frontal lobe function and episodic memory. Frontal lobe damage results in impaired goal setting, and impaired selection, monitoring, and verification of responses. It can be predicted that memory functions that engage these processes will be selectively impaired in patients with damage to the frontal lobe. PET studies from his group indicate that the right and left frontal lobes are predominantly activated by episodic encoding and retrieval tasks respectively. This lateralised activation seems to reflect the use of frontal executive processes in these tasks. In particular left frontal activation may reflect semantic organisational processes involved in encoding, whereas right frontal activation reflects the use of organisational strategies during monitored search and retrieval operations.
The afternoon session was devoted to clinical controversies and the keynote lecture on the treatment of memory disorders was given by Dr Barbara Wilson (Cambridge). Although it is impossible to restore lost memory function much can be done to bypass problems and compensate difficulties. She presented data suggesting that in patients with loss of episodic memory errorless learning (avoiding making mistakes during learning) is preferable to trial and error learning and emphasised the need to consider coexisting cognitive and emotional problems that may contribute to the overall disability. She went on to describe the use of external memory aids and the increasing role that new technology (for example, computers, pagers, "smart" houses) is likely to play in the future.

Dr Narinder Kapur (Wessex) gave a talk entitled focal retrograde amnesia in neurological disease: fact or fiction? Focal retrograde amnesia refers to pronounced loss of memory for preinjury/preillness events and knowledge with relatively preserved retention of new information. He concluded, after reviewing his own cases, that patients with focal retrograde amnesia fall into three different categories: (1) Those with severe brain pathology and no psychological precipitants. Anteroinferior, rather than medial temporal regions, seem to bear the brunt of the damage in these patients, although in some patients frontal lobe pathology has also been implicated; (2) those in whom organic and psychological factors coexist, the second being responsible for part or all of the retrograde amnesia; and (3) those in whom only psychological factors are present.

Dr Adam Zeman (Edinburgh) reviewed transient amnesic syndromes. Transient global amnesia gives rise to a dense anterograde amnesia and variable retrograde amnesia with otherwise normal cognitive function lasting for several hours. Functional neuroimaging studies suggest that temporal lobe dysfunction is commonly responsible for transient global amnesia, although the thalami and frontal lobes may be implicated. Current research suggests that transient global amnesia may be loosely related to migraine and that a process akin to the spreading depression of Leao may be responsible. A subset of patients with transient global amnesia prove to have temporal lobe epilepsy and some have postulated the existence of "transient epileptic amnesia". Transient epileptic amnesia is briefer and more likely to recur that transient global amnesia, often occurs on waking, and responds to anticonvulsants. It remains to be determined whether transient epileptic amnesia is an ictal or postictal phenomenon, and the cause of the patchy but persistent retrograde amnesia seen in some of these patients needs further study.

Professor John Morton (London) referred to the controversy surrounding recovered memories/false memories emphasising that this is largely political, clinical, or forensic rather than scientific, as it is possible for people to believe that certain things happen to them when they did not and vice versa. He put forward the concept 
of "headed records" whereby a chunk of information or "record" is laid down with a particular "heading" or retrieval cue. Duplicate records may have different headings and the same heading may contain two different records. This framework may be useful in explaining how memories may be lost or recovered.

The meeting closed with a critique of the day's papers by Dr Michael Kopelman (London) who put forward the case against fractionating memory to smithereens.
A lively debate followed with the participation of all the speakers.

The summer meeting of the BNPA will be the third joint meeting with the American Neuropsychiatric Association (ANPA) and will take place on 20-22 July at Robinson College, Cambridge.

MARIA A RON The National Hospital for Neurology and Neurosurgery, Queen Square,

政

